\title{
Continuous observer design for a class of multi-output nonlinear systems with multi-rate sampled and delayed output measurements
}

\author{
Yanjun Shen ${ }^{1,}$, Daoyuan Zhang ${ }^{\text {a }}$, Xiaohua Xia ${ }^{b}$ \\ ${ }^{a}$ Hubei Provincial Collaborative Innovation Center for New Energy Microgrid, \\ China Three Gorges University, Yichang, Hubei, 443002, China \\ ${ }^{\mathrm{b}}$ Department of Electrical, Electronic and Computer Engineering, \\ University of Pretoria, Pretoria 0002, South Africa
}

\begin{abstract}
In this paper, continuous observer is designed for a class of multi-output nonlinear systems with multi-rate sampled and delayed output measurements. The time delay may be larger or less than the sampling intervals. The sampled and delayed measurements are used to update the observer whenever they are available. Sufficient conditions are presented to ensure global exponential stability of the observation errors by constructing a Lyapunov-Krasovskii function. A numerical example is given to illustrate the effectiveness of the proposed methods.
\end{abstract}

Key words: hybrid systems, continuous observer, multi-rate, delayed measurements

\section{Introduction}

Recently, the problem of design global convergent observers for nonlinear systems has made great progress. For the observation of nonlinear systems, one can use extended Luenberger observers (Zeitz, 1987), normal form observers (Xia and Gao, 1988; Xia and Gao, 1989; Bestle and Zeitz, 1983; Krener and Isidori, 1983), Lyapunov based observers (Raghavan and Hedrick, 1994; Thau, 1973), high-gain observers (Gauthier, Hammouri and Othman, 1992; Gauthier and Kupka, 1994), sliding mode observers (Haskara, Özgüner and Utkin, 1998) and moving horizon/optimization based observers (Michalska and Mayne, 1995). Among these methods, high-gain observers play an important role and can be used to a large class of nonlinear systems with a triangular structure after a coordinate change. New developments of high gain observers have been carried out in various directions (Gauthier, Hammouri and Othman, 1992; Praly, 2003; Deza, 1991; Deza, Bossanne, Busvelle, Gauthier and Rakotopara, 1993; Andrieu, Praly and Astolfi, 2009). For example, the result of (Gauthier, Hammouri and Othman, 1992) is extended to a class of nonlinear systems where the non-

\footnotetext{
1 Corresponding author
}

linear terms admit an incremental rate depending on the measured output (Praly, 2003). In (Deza, 1991), the authors considered observer design for multi-input and multi-output (MIMO) nonlinear systems. The result has been extended to a class of MIMO nonlinear systems, in which interconnection between the blocks are not allowed (Deza, Bossanne, Busvelle, Gauthier and Rakotopara, 1993). Based on the observer normal form, another extension for the multi-output systems has been studied in (Rudolph and Zeitz, 1994). However, the nonlinearity of each block does not allow the unmeasurable states of its own block. Under the conditions of observability and triangular structure, a nonlinear system can be transformed into the block low triangular form considered in (Shim, Son and Seo, 2001) by a coordinate transformation. Then, semi-global observer has been designed for nonlinear systems with interconnections between the subsystems (Shim, Son and Seo, 2001). The nonlinear system with block lower triangular form is rather general when nonlinear changes of coordinates are allowed. It includes the control-affine multi-input and single-output (MISO) nonlinear systems which are strongly observable for any input (Gauthier, Hammouri and Othman, 1992) and the control-affine MIMO nonlinear systems which are strongly observable for any input for each output taken separately (Deza, 1991). 
Moreover, it can be used to express some physical systems. For example, the dynamical equations of a permanent magnet stepper motor can be transformed into the block lower triangular form (Mahmoud and Khalil, 2002). The estimation errors can converge to the origin in finite-time by using high gain observers in conjunction with applications of geometric homogeneity and Lyapunov theories (Shen and Xia, 2008; Shen and Huang, 2009; Li, Xia and Shen, 2013).

It should be noted that the above results on observer design are based on continuous-time analysis. However, for a networked control system, the output is only available at discrete-time instants since it is usually transmitted through a shared band-limited digital communication network. Therefore, observer design for continuous systems with sampled and delayed output measurements has attracted the control community wide attention. There exist three main approaches to design observer for continuous systems with sampled and delayed measurements, for example, discrete time analysis based on a discretized model (Arcak and Nešić, 2004; Barbot, Monaco and Normand-Cyrot, 1999; Nešić, Teel and Kokotović, 1999), continuous time analysis followed by discretization (Khalil, 2004; Nešić and Teel, 2004; Postoyan and Nešić, 2012; Wang, Nešić and Postoyan, 2015), and a mixed continuous and discrete time analysis without discretization (Deza, Busvelle, Gauthier and Rakotopora, 1992; AhmedAli and Lamnabhi-Lagarrigue, 2012; Raff, Kögel and Allgöwer, 2008; Van Assche, Ahmed-Ali, Ham and Lamnabhi-Lagarrigue, 2011; Nadri, Hammouri and Grajales, 2013; Karafyllis and Kravaris, 2009; AhmedAli, Van Assche, Massieu and Dorléans, 2013; Zhang, Shen and Xia, 2014). More specifically, two classes of global exponential observers have been presented for a class of continuous systems with sampled and delayed measurements in (Ahmed-Ali, Van Assche, Massieu and Dorléans, 2013). By using the same methods, exponential convergent observers were proposed for nonlinear systems with sampled and delayed measurements in (Ahmed-Ali, Karafyllis and LamnabhiLagarrigue, 2013). The observers designed in (AhmedAli, Van Assche, Massieu and Dorléans, 2013; AhmedAli, Karafyllis and Lamnabhi-Lagarrigue, 2013) are in essence discontinuous. The authors in (Zhang, Shen and Xia, 2014) proposed a continuous observer for a class of nonlinear systems with sampled and delayed measurements based on an auxiliary integral technique. But there is a constraint condition on time delay, that is the maximum delay must be less than the minimum sampling interval as in (Ahmed-Ali, Van Assche, Massieu and Dorléans, 2013; Ahmed-Ali, Karafyllis and Lamnabhi-Lagarrigue, 2013).

In this paper, we address continuous observer design for a class of multi-output nonlinear systems with multi-rate sampled and delayed output measurements. The considered nonlinear systems are in continuous time while the outputs are in discrete time. In order to overcome the difficulties in analysis, we represent the sampled-data system as a continuous time system with successive delay components by some transformations. The time delays are more general than those in (Ahmed-Ali, Van Assche, Massieu and Dorléans, 2013; Ahmed-Ali, Karafyllis and Lamnabhi-Lagarrigue, 2013; Zhang, Shen and Xia, 2014) since they may be larger or smaller than the sampling periods. Our main contributions include: a) Continuous observer is designed for a class of multi-output nonlinear systems whenever the sampled and delayed measurements are available. b) The observer is transformed into a continuous nonlinear system with time-varying delay by time delay method. Then, by constructing a Lyapunov-Krasovskii function, sufficient conditions are presented to ensure that the observation errors are globally exponentially stabile. c) Different high gains are used to dominate the nonlinear terms in each block. Then, upper bounds on each sampling period and time delay are also achieved.

This paper is organized as follows. In Section 2, continuous observers are presented for a class of multi-output nonlinear systems with multi-rate sampled and time delayed measurements. In Section 3, an example is used to illustrate the validity of the proposed design methods. Finally, Section 4 concludes the paper.

Throughout this paper, let $\mathbb{R}^{n}$ denote $n$-dimension real space, $I$ denote an identity matrix, $\operatorname{diag}\{\}$ denote a diagonal matrix, and the superscript "T" stand for matrix transposition. For any $x \in \mathbb{R}^{n}$, let $\|x\|=\left(x^{\top} x\right)^{1 / 2}$. For a continuous function $f: \mathbb{R} \rightarrow \mathbb{R}$ and $t \in \mathbb{R}$, let $\lim _{s \rightarrow t^{-}} f(s)=\lim _{s \rightarrow t, s<t} f(s) . \lambda_{\max }(P)$ and $\lambda_{\min }(P)$ denote the largest and the smallest eigenvalues of $P \in$ $\mathbb{R}^{n \times n}$, respectively.

\section{Main results}

In this section, we consider the following multi-output nonlinear systems

$$
\left\{\begin{array}{l}
\dot{x}(t)=A x(t)+B(x(t), u(t)), \\
y(t)=C x(t)=\left[C_{1} x^{1}(t), \cdots C_{m} x^{m}(t)\right]^{\top},
\end{array}\right.
$$

where the state $x(t) \in \mathbb{R}^{n}$, the input $u(t) \in \mathbb{R}^{p}$, the output $y(t) \in \mathbb{R}^{m}, x(t)=\left[x^{1}(t)^{\top}, \cdots, x^{m}(t)^{\top}\right]^{\top}$, $x^{i}(t) \in \mathbb{R}^{\lambda_{i}}(1 \leq i \leq m)$ is the $i$ th partition of the state $x(t) ; A=\operatorname{diag}\left\{A_{1}, \cdots, A_{m}\right\}, A_{i}$ is $\lambda_{i} \times \lambda_{i}$ matrix of Brunovsky form, that is $A_{i}=\left[\begin{array}{cccc}0 & 1 & \cdots & 0 \\ \vdots & \vdots & \ddots & \vdots \\ 0 & 0 & \cdots & 1 \\ 0 & 0 & \cdots & 0\end{array}\right]$, $C=\operatorname{diag}\left\{C_{1}, \cdots, C_{m}\right\}, C_{i}=[1,0, \cdots, 0]_{1 \times \lambda_{i}}$, and 
$B(x(t), u(t))=\left[b^{1}(x(t), u(t))^{\top}, \cdots, b^{m}(x(t), u(t))^{\top}\right]^{\top}$ in which the $j$ th element of $b^{i}(\cdot), b_{j}^{i}(\cdot)$ has the following structural dependence on the states:

$b_{j}^{i}(t)=b_{j}^{i}\left(x^{1}(t), \cdots, x^{i-1}(t) ; x_{1}^{i}(t), \cdots, x_{j}^{i}(t) ; u(t)\right)$,

for all $1 \leq i \leq m$ and $1 \leq j \leq \lambda_{i}$. Thus, $b_{j}^{i}$ is independent of the lower states $\left(x_{j+1}^{i}, \cdots, x_{\lambda_{i}}^{i}\right)$ of the $i$ th block and the states of the lower blocks $\left(x^{i+1}, \cdots, x^{m}\right)$. The $i$ th block of the above system can be expressed as follows

$$
\left\{\begin{array}{l}
\dot{x}_{1}^{i}(t)=x_{2}^{i}(t)+b_{1}^{i}\left(x(t)^{[1, i-1]} ; x_{1}^{i}(t) ; u(t)\right), \\
\vdots \\
\dot{x}_{\lambda_{i}-1}^{i}(t)=x_{\lambda_{i}}^{i}(t) \\
\quad+b_{\lambda_{i}-1}^{i}\left(x(t)^{[1, i-1]} ; x(t)_{\left[1, \lambda_{i}-1\right]}^{i} ; u(t)\right), \\
\dot{x}_{\lambda_{i}}^{i}(t)=b_{\lambda_{i}}^{i}\left(x(t)^{[1, i-1]} ; x(t)_{\left[1, \lambda_{i}\right]}^{i} ; u(t)\right)
\end{array}\right.
$$

where $x_{j}^{i}(t)$ is the $j$ th element of the $i$ th block $x^{i}(t)$. The abbreviation $x(t)^{[1, k]}:=\left[x^{1}(t)^{\top}, \cdots, x^{k}(t)^{\top}\right]^{\top}$ and $x(t)_{[1, j]}^{i}:=\left[x_{1}^{i}(t), \cdots, x_{j}^{i}(t)\right]^{\top}$ can be used to simplify the notation. We assume that there are $m$ sensors in $m$ channels to sample the output $y$ at sampling instants $t_{k}^{i}$, and $t_{k}^{i}<t_{k+1}^{i}(i=1, \cdots, m$ and $k=0,1,2, \cdots, \infty)$, where $\left\{t_{k}^{i}\right\}(i=1, \cdots, m)$ are strictly increasing sequences and satisfy that $\lim _{k \rightarrow \infty} t_{k}^{i}=\infty$. The sampled measures are available at instants $t_{k}^{i}+\tau_{k}^{i}(i=1, \cdots, m)$, where $\tau_{k}^{i}>0$ $(i=1, \cdots, m)$ denote the transmission delay, which are unknown but have an upper bound $\bar{\tau}_{i}$. The nonlinear terms $b_{j}^{i}(\cdot)$ are assumed to satisfy the following global Lipschitz conditions with Lipschitz constant $l_{1}>0$,

$$
\begin{gathered}
\mid b_{j}^{i}\left(x^{1}, \cdots, x^{i-1} ; x_{1}^{i}, \cdots, x_{j}^{i} ; u\right)-b_{j}^{i}\left(\hat{x}^{1}, \cdots, \hat{x}^{i-1} ; \hat{x}_{1}^{i},\right. \\
\left.\cdots, \hat{x}_{j}^{i} ; u\right) \mid \leq l_{1}\left(\left|x_{1}^{1}-\hat{x}_{1}^{1}\right|+\left|x_{2}^{1}-\hat{x}_{2}^{1}\right|+\cdots\left|x_{j}^{i}-\hat{x}_{j}^{i}\right|\right) \\
1 \leq i \leq m, 1 \leq j \leq \lambda_{i} .
\end{gathered}
$$

Now, the explicit form of the $i$ th block of the observer is given as follows:

$$
\left\{\begin{array}{c}
\dot{\hat{x}}_{1}^{i}(t)=\hat{x}_{2}^{i}(t)+L_{i} a_{1}^{i} e_{1}^{i}\left(t_{k}^{i}\right) \\
\quad+b_{1}^{i}\left(\hat{x}(t)^{[1, i-1]} ; \hat{x}_{1}^{i}(t) ; u(t)\right), \\
\vdots \\
\dot{\hat{x}}_{\lambda_{i}-1}^{i}(t)=\hat{x}_{\lambda_{i}}^{i}(t)+L_{i}^{\lambda_{i}-1} a_{\lambda_{i}-1}^{i} e_{1}^{i}\left(t_{k}^{i}\right) \\
\quad+b_{\lambda_{i}-1}^{i}\left(\hat{x}(t)^{[1, i-1]} ; \hat{x}(t)_{\left[1, \lambda_{i}-1\right]}^{i} ; u(t)\right), \\
\quad+b_{\lambda_{i}}^{i}\left(\hat{x}(t)^{[1, i-1]} ; \hat{x}(t)_{\left[1, \lambda_{i}\right]}^{i} ; u(t)\right), \\
\hat{x}_{\lambda_{i}}^{i}(t)=L_{\lambda_{i}}^{\lambda_{i}} e_{1}^{i}\left(t_{k}^{i}\right) \\
\hat{x}_{j}^{i}\left(t_{k+1}^{i}+\tau_{k+1}^{i}\right)=\lim _{t \rightarrow t_{k+1}^{i}+\tau_{k+1}^{i}-\hat{x}_{j}^{i}(t),} \\
j=1,2, \cdots, \lambda_{i}, t \in\left[t_{k}^{i}+\tau_{k}^{i}, t_{k+1}^{i}+\tau_{k+1}^{i}\right), k \geq 0
\end{array}\right.
$$

where $\hat{x}_{j}^{i}(t)=\hat{x}_{j_{0}}^{i}$ for $t \in\left[t_{0}, t_{0}+\tau_{0}^{i}\right]\left(t_{0}=t_{0}^{i}\right), i=$ $1, \cdots, m$ and $j=1, \cdots, \lambda_{i}, e_{1}^{i}\left(t_{k}^{i}\right)=x_{1}^{i}\left(t_{k}^{i}\right)-\hat{x}_{1}^{i}\left(t_{k}^{i}\right)$, $L_{i} \geq 1$ and $a_{j}^{i}\left(1 \leq i \leq m, 1 \leq j \leq \lambda_{i}\right)$ are positive real numbers, and will be given later. The definition of global exponential stable observer for the system (2) is given as follows.

Definition 1 We say that the system (4) is a global exponential stable observer for the system (2), if there exist a non-decreasing function $N: \mathbb{R}^{+} \times \mathbb{R}^{+} \rightarrow \mathbb{R}^{+}$ and a positive constant $\kappa$ such that $\|\hat{x}(t)-x(t)\| \leq$ $\exp \left(-\kappa\left(t-t_{0}\right)\right) N\left(\left\|x_{0}\right\|,\left\|\hat{x}_{0}\right\|\right)$ for any $x_{0} \in \mathbb{R}^{n}, \hat{x}_{0} \in \mathbb{R}^{n}$.

Remark 1 The outputs $y_{i}(i=1, \cdots, m)$ are transmitted through $m$ channels, respectively. We can use $m$ sensors to detect them. Therefore, although $\tau_{k}^{i}$ are unknown, we can obtain the instant that the sampled data at instants $t_{k}^{i}$ is available. In other word, $e_{1}^{i}\left(t_{k}^{i}\right)$ is updated automatically whenever the sampled and delayed measurement $y_{i}\left(t_{k}^{i}\right)$ arrives.

From (2) and (4), the dynamics of the state error can be obtained

$$
\left\{\begin{array}{c}
\dot{e}_{1}^{i}(t)=e_{2}^{i}(t)-L_{i} a_{1}^{i} e_{1}^{i}(t)+L_{i} a_{1}^{i} \int_{t_{k}^{i}}^{t} \dot{e}_{1}^{i}(s) d s+\tilde{b}_{1}^{i}, \\
\vdots \\
\dot{e}_{\lambda_{i}-1}^{i}(t)=e_{\lambda_{i}}^{i}(t)-L_{i}^{\lambda_{i}-1} a_{\lambda_{i}-1}^{i} e_{1}^{i}(t) \\
\quad+L_{i}^{\lambda_{i}-1} a_{\lambda_{i}-1}^{i} \int_{t_{k}^{i}}^{t} \dot{e}_{1}^{i}(s) d s+\tilde{b}_{\lambda_{i}-1}^{i}, \\
\dot{e}_{\lambda_{i}}^{i}(t)=-L_{i}^{\lambda_{i}} a_{\lambda_{i}}^{i} e_{1}^{i}(t)+L_{i}^{\lambda_{i}} a_{\lambda_{i}}^{i} \int_{t_{k}^{i}}^{t} \dot{e}_{1}^{i}(s) d s+\tilde{b}_{\lambda_{i}}^{i}, \\
e_{j}^{i}\left(t_{k+1}^{i}+\tau_{k+1}^{i}\right)=\lim _{t \rightarrow t_{k+1}^{i}+\tau_{k+1}^{i}-e_{j}^{i}(t),} \\
j=1, \cdots, \lambda_{i}, t \in\left[t_{k}^{i}+\tau_{k}^{i}, t_{k+1}^{i}+\tau_{k+1}^{i}\right), k \geq 0,
\end{array}\right.
$$

where $e=\left[e^{1}(t)^{\top}, \cdots, e^{m}(t)^{\top}\right]^{\top}, e^{i}(t)=\left[e_{1}^{i}(t), \cdots, e_{\lambda_{i}}^{i}(t)\right]^{\top}$, $e_{j}^{i}(t)=x_{j}^{i}(t)-\hat{x}_{j}^{i}(t), \tilde{b}_{j}^{i}=b_{j}^{i}\left(x(t)^{[1, i-1]} ; x(t)_{[1, j]}^{i} ; u(t)\right)$ $-b_{j}^{i}\left(\hat{x}(t)^{[1, i-1]} ; \hat{x}(t)_{[1, j]}^{i} ; u(t)\right),\left(1 \leq i \leq m, 1 \leq j \leq \lambda_{i}\right)$.

Remark 2 Note that $\lim _{t \rightarrow t_{k+1}^{i}+\tau_{k+1}^{i}} e_{j}^{i}(t)=e_{j}^{i}\left(t_{k+1}^{i}+\right.$ $\left.\tau_{k+1}^{i}\right)$, then $e^{i}(t)$ is continuous on $\left[t_{k}^{i}+\tau_{k}^{i}, t_{k+1}^{i}+\tau_{k+1}^{i}\right]$. On the other hand, the evolution process $e_{1}^{i}\left(t_{k}^{i}\right)=$ $x_{1}^{i}\left(t_{k}^{i}\right)-\hat{x}_{1}^{i}\left(t_{k}^{i}\right)$ is updated at instants $t_{k}^{i}+\tau_{k}^{i}$, whereas the sampled measurement $y_{i}(t)$ is sampled at instants $t_{k}^{i}$. Therefore, the system (5) is continuous, delayed and hybrid in nature. Similar systems have been investigated in (Karafyllis, 2007a; Karafyllis, 2007b; Karafyllis and Jiang, 200\%; Ahmed-Ali, Van Assche, Massieu and Dorléans, 2013).

Let $\eta_{i}(t)=t-t_{k}^{i}$. Then, $t_{k}^{i}$ in (5) can been expressed by

$t_{k}^{i}=t-\eta_{i}(t)$

Therefore, $0<\eta_{i}(t)=t-t_{k}^{i} \leq t_{k+1}^{i}+\tau_{k+1}^{i}-t_{k}^{i}<h_{i}$, where $h_{i}>0$. Our aim is to find the bounds of $h_{i}$ such 
that the error system (5) is globally exponentially stable.

Consider the following change of coordinates $\varepsilon_{j}^{i}=$ $\frac{e_{j}^{i}}{L_{i}^{\lambda_{j}^{i}-1}}, \quad 1 \leq i \leq m, 1 \leq j \leq \lambda_{i}$, where $\lambda_{j}^{i}=\Sigma_{k=1}^{i-1} \lambda_{k}+j$, $\left(1 \leq i \leq m, 1 \leq j \leq \lambda_{i}\right)$. Then,

$$
\left\{\begin{array}{c}
\dot{\varepsilon}_{1}^{i}(t)=L_{i} \varepsilon_{2}^{i}(t)-L_{i} a_{1}^{i} \varepsilon_{1}^{i}(t) \\
\quad+L_{i} a_{1}^{i} \int_{t-\eta_{i}(t)}^{t} \dot{\varepsilon}_{1}^{i}(s) d s+\frac{\tilde{b}_{1}^{i}}{L_{i}^{\lambda_{1}^{i}-1}} \\
\vdots \\
\dot{\varepsilon}_{\lambda_{i}-1}^{i}(t)=L_{i} \varepsilon_{\lambda_{i}}^{i}(t)-L_{i} a_{\lambda_{i}-1}^{i} \varepsilon_{1}^{i}(t) \\
\quad+L_{i} a_{\lambda_{i}-1}^{i} \int_{t-\eta_{i}(t)}^{t} \dot{\varepsilon}_{1}^{i}(s) d s+\frac{\tilde{b}_{\lambda_{i}-1}^{i}}{L_{i}^{\lambda_{\lambda_{i}-1}-1}}, \\
\dot{\varepsilon}_{\lambda_{i}}^{i}(t)=-L_{i} a_{\lambda_{i}}^{i} \varepsilon_{1}^{i}(t)+L_{i} a_{\lambda_{i}}^{i} \int_{t-\eta_{i}(t)}^{t} \dot{\varepsilon}_{1}^{i}(s) d s \\
\quad+\frac{\tilde{b}_{\lambda_{i}}^{i}}{L_{i}^{\lambda_{\lambda_{i}}^{i}-1}}, \quad i=1, \cdots, m .
\end{array}\right.
$$

Remark $3 t_{k}^{i}$ denotes the sampling instant, and $\eta_{i}(t)$ is a time-varying delay with bound $h_{i}$. The transformation (6) is used to represent the error system (5) as a continuous time system (7) with successive delay components. The problem of continuous observer design can be solved based on this model.

Now, we give the following result for the system (2).

Theorem 1 Consider the system (2) with the condition (3). If $L_{i}$ satisfy $L_{i}>\max \left\{1, l_{1}, 8 \lambda_{\lambda_{i}}^{i} l_{1} \bar{p}_{2}^{i}, L_{i-1}\right\}$, and $a_{j}^{i}>0\left(1 \leq i \leq m, 1 \leq j \leq \lambda_{i}\right)$ are given such that there exists a symmetric positive definite matrix $P$ such that

$\bar{A}^{\top} P+P \bar{A} \leq-I$,

and

$$
\begin{gathered}
h_{i}<\min \left\{\frac{1}{4 L_{i}\left(3+\lambda_{1}^{i}\right)^{2}\left(1+a_{1}^{i 2}\right)}, \frac{1}{16 L_{i} \lambda_{i} \bar{\lambda}_{i}^{2} \bar{a}_{i}},\right. \\
\left.\frac{1}{2 L_{i} \sqrt{\left(3+\lambda_{1}^{i}\right)} a_{1}^{i}}\right\}, i=1, \cdots, m,
\end{gathered}
$$

then, the system (4) is a global exponential stable observer for the system (2), where $L_{0} \geq 1$, $\bar{A}=\operatorname{diag}\left\{\bar{A}_{1}, \cdots, \bar{A}_{m}\right\}, P=\operatorname{diag}\left\{P_{1}, \cdots, P_{m}\right\}, \underline{\lambda}_{i}=$ $\lambda_{\min }\left(P_{i}\right), \bar{\lambda}_{i}=\lambda_{\max }\left(P_{i}\right), \bar{\lambda}=\max _{\{1 \leq i \leq m\}}\left\{\bar{\lambda}_{i}\right\}, \bar{a}_{i}=$ $\max _{\left\{1 \leq j \leq \lambda_{i}\right\}}\left\{\left(a_{j}^{i}\right)^{2}\right\}, \bar{p}_{2}^{i}=\max _{\left\{1 \leq j \leq \lambda_{i}, 1 \leq r \leq \lambda_{i}\right\}}\left\{\left|P_{j, r}^{i}\right|\right\}$, $(1 \leq i \leq m), P_{j, r}^{i}$ is the element of $P_{i}$ at the $j$ th line and rth column, and $\bar{A}_{i}=\left[\begin{array}{cccc}-a_{1}^{i} & 1 & \cdots & 0 \\ \vdots & \vdots & \ddots & \vdots \\ -a_{\lambda_{i}-1}^{i} & 0 & \cdots & 1 \\ -a_{\lambda_{i}}^{i} & 0 & \cdots & 0\end{array}\right]$.
Proof: Consider the positive definite function

$V_{1}(t)=\varepsilon(t)^{\top} P \varepsilon(t)=\sum_{i=1}^{m} \varepsilon^{i}(t)^{\top} P_{i} \varepsilon^{i}(t)$,

where $\varepsilon(t)=\left[\varepsilon^{1}(t)^{\top}, \cdots, \varepsilon^{m}(t)^{\top}\right]^{\top}, \varepsilon^{i}(t)=\left[\varepsilon_{1}^{i}(t), \cdots\right.$, $\left.\varepsilon_{\lambda_{i}}^{i}(t)\right]^{\top},(1 \leq i \leq m)$. Then, the derivative of $V_{1}(t)$ along the system $(7)$ is given by

$$
\begin{gathered}
\left.\quad \frac{d}{d t} V_{1}(t)\right|_{(7)}=\sum_{i=1}^{m} L_{i} \varepsilon^{i}(t)^{\top}\left(\bar{A}_{i}^{\top} P_{i}+P_{i} \bar{A}_{i}\right) \varepsilon^{i}(t) \\
+2 \sum_{i=1}^{m} L_{i}\left(a_{1}^{i}, a_{2}^{i}, \cdots, a_{\lambda_{i}}^{i}\right)\left(\int_{t-\eta_{i}(t)}^{t} \dot{\varepsilon}_{1}^{i}(s) d s\right) P_{i} \varepsilon^{i}(t) \\
+2 \sum_{i=1}^{m} \sum_{r=1}^{\lambda_{i}} \sum_{j=1}^{\lambda_{i}} \frac{\tilde{b}_{j}^{i}}{L_{i}^{\lambda_{j}^{i}-1}} \varepsilon_{j}^{i}(t) P_{j, r}^{i} \\
\leq-\sum_{i=1}^{m} L_{i} \varepsilon^{i}(t)^{\top} \varepsilon^{i}(t)+\frac{1}{4} \sum_{i=1}^{m} L_{i} \varepsilon^{i}(t)^{\top} \varepsilon^{i}(t) \\
+4 \sum_{i=1}^{m} L_{i}\left(a_{1}^{i}, a_{2}^{i}, \cdots, a_{\lambda_{i}}^{i}\right) \\
\times \int_{t-\eta_{i}(t)}^{t} \dot{\varepsilon}_{1}^{i}(s) d s P_{i} P_{i}\left(a_{1}^{i}, a_{2}^{i}, \cdots, a_{\lambda_{i}}^{i}\right)^{\top} \int_{t-\eta_{i}(t)}^{t} \dot{\varepsilon}_{1}^{i}(s) d s \\
+2 l_{1} \sum_{i=1}^{m} \sum_{r=1}^{\lambda_{i}} \sum_{j=1}^{\lambda_{i}}\left|\varepsilon_{j}^{i}(t) P_{j, r}^{i}\right| \\
\quad \times\left(\sum_{k=1}^{j} \sum_{a=1}^{\lambda_{b}} \sum_{b=1}^{i-1}\left|\varepsilon_{a}^{b}\right|+\left|\varepsilon_{k}^{i}\right|\right) \\
\leq-\sum_{i=1}^{m}\left(\frac{3}{4} L_{i}-2 \lambda_{\lambda_{i}}^{i} l_{1} \bar{p}_{2}^{i}\right) \varepsilon^{i}(t)^{\top} \varepsilon^{i}(t) \\
+4 \sum_{i=1}^{m} L_{i} \lambda_{i} \bar{\lambda}_{i}^{2} \bar{a}_{i}\left(\int_{t-\eta_{i}(t)}^{t} \dot{\varepsilon}_{1}^{i}(s) d s\right)^{2} .
\end{gathered}
$$

Note that $L_{i}>\left\{8 \lambda_{\lambda_{i}}^{i} l_{1} \bar{p}_{2}^{i}\right\}$. Then, we have

$$
\begin{aligned}
& \left.\frac{d}{d t} V_{1}(t)\right|_{(7)} \leq-\frac{1}{2} \sum_{i=1}^{m} L_{i} \varepsilon^{i}(t)^{\top} \varepsilon^{i}(t) \\
& +4 \sum_{i=1}^{m} L_{i} \lambda_{i} \bar{\lambda}_{i}^{2} \bar{a}_{i}\left(\int_{t-\eta_{i}(t)}^{t} \dot{\varepsilon}_{1}^{i}(s) d s\right)^{2} .
\end{aligned}
$$

By Lemma 1 in $(\mathrm{Gu}, 2000)$, we have

$\left|\int_{t-\eta_{i}(t)}^{t} \dot{\varepsilon}_{1}^{i}(s) d s\right|^{2} \leq h_{i} \int_{t-h_{i}}^{t} \dot{\varepsilon}_{1}^{i}(s)^{2} d s$.

It follows from (10) and (11) that

$$
\begin{aligned}
& \left.\frac{d}{d t} V_{1}(t)\right|_{(7)} \leq-\frac{1}{2} \sum_{i=1}^{m} L_{i} \varepsilon^{i}(t)^{\top} \varepsilon^{i}(t) \\
& +4 \sum_{i=1}^{m} L_{i} \lambda_{i} \bar{\lambda}_{i}^{2} \bar{a}_{i} h_{i} \int_{t-h_{i}}^{t} \dot{\varepsilon}_{1}^{i}(s)^{2} d s .
\end{aligned}
$$

Consider the following auxiliary integral function

$V_{2}(t)=\sum_{i=1}^{m} \int_{t-h_{i}}^{t} \int_{\rho}^{t} \dot{\varepsilon}_{1}^{i}(s)^{2} d s d \rho, t \geq t_{0}+\bar{h}$

where $\bar{h}=\max _{1 \leq i \leq m}\left\{h_{i}\right\}$. We have,

$$
\begin{aligned}
& \frac{d V_{2}(t)}{d t} \leq \sum_{i=1}^{m} L_{i}^{2}\left(3+\lambda_{1}^{i}\right)^{2}\left(1+a_{1}^{i}{ }^{2}\right) h_{i} \varepsilon^{i}(t)^{T} \varepsilon^{i}(t) \\
& +\sum_{i=1}^{m} L_{i}^{2}\left(3+\lambda_{1}^{i}\right) a_{1}^{i^{2}} h_{i}^{2} \int_{t-h_{i}}^{t} \dot{\varepsilon}_{1}^{i}(s)^{2} d s \\
& \quad-\sum_{i=1}^{m} \int_{t-h_{i}}^{t} \dot{\varepsilon}_{1}^{i}(s)^{2} d s, t \geq t_{0}+\bar{h}
\end{aligned}
$$


and

$V_{2}(t) \leq \sum_{i=1}^{m} h_{i} \int_{t-h_{i}}^{t} \dot{\varepsilon}_{1}^{i}(s)^{2} d s$

Construct the following Lyapunov-Krasovskii function

$V(t)=V_{1}(t)+V_{2}(t), t \geq t_{0}+\bar{h}$.

From (12), (13) and (9), we have

$$
\begin{gathered}
\left.\frac{d V(t)}{d t}\right|_{(7)} \leq-\sum_{i=1}^{m}\left(\frac{1}{2}-\left(3+\lambda_{1}^{i}\right)^{2}\left(1+a_{1}^{i}{ }^{2}\right) h_{i} L_{i}\right) \\
\times L_{i} \varepsilon^{i}(t)^{\top} \varepsilon^{i}(t)+\sum_{i=1}^{m}\left(4 L_{i} \lambda_{i} \bar{\lambda}_{i}^{2} \bar{a}_{i} h_{i}\right. \\
\left.+\left(3+\lambda_{1}^{i}\right) a_{1}^{i^{2}} h_{i}^{2} L_{i}^{2}-1\right) \int_{t-h_{i}}^{t} \dot{\varepsilon}_{1}^{i}(s)^{2} d s \\
\leq-\frac{L}{4 \lambda} V(t), t \geq t_{0}+\bar{h}
\end{gathered}
$$

where $\underline{L}=\min _{\{1 \leq i \leq m\}}\left\{L_{i}\right\}$. Then, $V(t) \leq \exp \left(-\frac{L}{4 \lambda}(t-\right.$ $\left.\left.t_{0}-\bar{h}\right)\right) V\left(t_{0}+\bar{h}\right), t \geq t_{0}+\bar{h}$. Since the nonlinear terms in the system (2) and (4) satisfy the global Lipschitz conditions (3), then, the solutions of (2) and (4) exist and are continuous on $\left[t_{0}, t_{0}+\bar{h}\right]$. Therefore, there exists a non-decreasing function $N: \mathbb{R}^{+} \times \mathbb{R}^{+} \rightarrow \mathbb{R}^{+}$such that $\|\hat{x}(t)-x(t)\| \leq \exp \left(-\frac{L}{4 \lambda}\left(t-t_{0}-\bar{h}\right)\right) N\left(\left\|x_{0}\right\|,\left\|\hat{x}_{0}\right\|\right)$ for any $x_{0} \in \mathbb{R}^{n}, \hat{x}_{0} \in \mathbb{R}^{n}$. Thus, the system (4) is a global exponential stable observer for the system (2).

\section{Numerical simulation}

In this section, we use an example to show the effectiveness of our high gain observer design for nonlinear systems with sampled and time delay measurements. Consider the following multi-output nonlinear system (Shim, Son and Seo, 2001):

$$
\left\{\begin{aligned}
\dot{x}_{1}(t)= & x_{2}(t)+0.01 u(t), \\
\dot{x}_{2}(t)= & -x_{1}(t)+0.1\left(1-x_{1}^{2}(t)\right) x_{2}(t)+0.1 x_{2}(t) u(t), \\
\dot{x}_{3}(t)= & x_{4}(t)+0.01 x_{2}(t) x_{3}(t) \exp (u(t)), \\
\dot{x}_{4}(t)= & -x_{3}(t)+0.1\left(1-x_{3}^{2}(t)\right) x_{4}(t) \\
& +\frac{1}{1+\left(x_{2}(t) x_{4}(t)\right)^{2}} u(t), \\
y_{1}(t)= & x_{1}(t) \\
y_{2}(t)= & x_{3}(t),
\end{aligned}\right.
$$

where $x(t)=\left(x_{1}(t), x_{2}(t), x_{3}(t), x_{4}(t)\right)^{T}$, which is in the form of (1) with $m=2$ and $x^{1}(t)=\left(x_{1}(t), x_{2}(t)\right)^{T}$, and $x^{2}(t)=\left(x_{3}(t), x_{4}(t)\right)^{T}$. By $(4)$, the observer is given by

$$
\begin{aligned}
& \dot{\hat{x}}_{1}(t)=\hat{x}_{2}(t)+0.01 u(t)+3 L_{1}\left(y_{1}\left(t_{k}^{1}\right)-\hat{x}_{1}\left(t_{k}^{1}\right)\right), \\
& \dot{\hat{x}}_{2}(t)=-\hat{x}_{1}(t)+0.1\left(1-\hat{x}_{1}^{2}(t)\right) \hat{x}_{2}(t)+0.1 \hat{x}_{2}(t) u(t)
\end{aligned}
$$

$$
\begin{aligned}
& t \in\left[t_{k}^{1}+\tau_{k}^{1}, t_{k+1}^{1}+\tau_{k+1}^{1}\right), k \geq 0, \\
& \dot{\hat{x}}_{3}(t)=\hat{x}_{4}(t)+0.01 \hat{x}_{2}(t) \hat{x}_{3}(t) \exp (u(t)) \\
& \quad+2 L_{2}\left(y_{2}\left(t_{k}^{2}\right)-\hat{x}_{3}\left(t_{k}^{2}\right)\right), \\
& \quad \dot{\hat{x}}_{4}(t)=-\hat{x}_{3}(t)+0.1\left(1-\hat{x}_{3}^{2}(t)\right) \hat{x}_{4}(t) \\
& \quad+\frac{1}{1+\left(\hat{x}_{2}(t) \hat{x}_{4}(t)\right)^{2}} u(t)+L_{2}^{2}\left(y_{2}\left(t_{k}^{2}\right)-\hat{x}_{3}\left(t_{k}^{2}\right)\right), \\
& t \in\left[t_{k}^{2}+\tau_{k}^{2}, t_{k+1}^{2}+\tau_{k+1}^{2}\right), k \geq 0, \\
& \hat{x}_{i}\left(t_{k+1}^{2}+\tau_{k+1}^{2}\right)=\lim _{t \rightarrow t_{k+1}^{2}+\tau_{k+1}^{2}}-\hat{x}_{i}(t), i=3,4,
\end{aligned}
$$

where $t_{k}^{1}=k T_{1}-(1.1 \cdot$ rand $) T_{1}$ and $t_{k}^{2}=k T_{2}-(1.5$. rand) $T_{2}$, rand is a random number in the interval $[0,1]$, $\tau_{k}^{1}$ and $\tau_{k}^{2}$ denote the transmission delays, $T_{1}$ and $T_{2}$ are two positive real constants and will be given later. By simple computation, $P=\operatorname{diag}\left\{P_{1}, P_{2}\right\}$, where $P_{1}=$ $\left[\begin{array}{cc}0.8917 & -0.5695 \\ -0.5695 & 1.1735\end{array}\right], P_{2}=\left[\begin{array}{cc}0.5062 & -0.5052 \\ -0.5052 & 1.5124\end{array}\right]$. Then $\lambda_{\max }(P)=1.7223, \lambda_{\min }(P)=0.2963$. The other parameters are given as: $l=1.6, L_{1}=40, L_{2}=90$. $\tau_{k}^{1}$ and $\tau_{k}^{2}$ are simulated by random numbers in the interval $\left[0,1.5 T_{1}\right]$ and $\left[0,1.8 T_{2}\right]$. From the condition (9), we have $h_{1}=3.3 \times 10^{-5} \mathrm{~s}$ and $h_{2}=1.5 \times 10^{-5} \mathrm{~s}$. Let $T_{1}=1.0 \times 10^{-5} \mathrm{~s}$ and $T_{2}=0.5 \times 10^{-5} \mathrm{~s}$. Fig. 1 shows the simulation results with the initial condition of observer $\hat{x}(0)=[-10,-10,-10,-10]$.

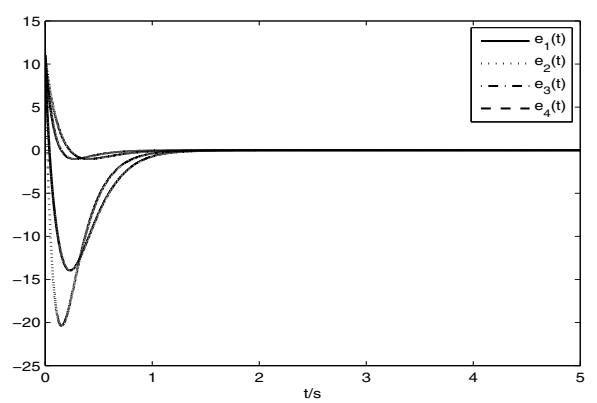

Fig. 1 Trajectories of the error states $e_{i}(t)(1 \leq i \leq 4)$ with $\hat{x}(0)$, $T_{1}=1.0 \times 10^{-5} s$ and $T_{2}=0.5 \times 10^{-5} s$.

\section{Conclusion}

In this paper, continuous observers were designed for a class of multi-output nonlinear systems with multirate sampled and delayed output measurements. The time delay might be larger or less than the sampling intervals. Sufficient conditions were presented to ensure global exponential stability of the observation errors by constructing a Lyapunov-Krasovskii function.

\section{Acknowledgments}

This work was supported by the National Science Foundation of China $(61374028,61273183)$, the Scientific In- 
novation Team Project of Hubei Provincial Department of Education (T201504).

\section{References}

T. Ahmed-Ali and F. Lamnabhi-Lagarrigue. (2012). High gain observer design for some networked control systems, IEEE Trans. Automat. Contr., 57(4), 995-1000.

T. Ahmed-Ali, V. Van Assche, J. Massieu and P. Dorléans. (2013). Continuous-discrete observer for state affine systems with sampled and delayed measurements, IEEE Trans. Automat. Contr., 58(4), 1085-1091.

T. Ahmed-Ali, I. Karafyllis and F. Lamnabhi-Lagarrigue. (2013). Global exponential sampled-data observers for nonlinear systems with delayed measurements, Syst. Control Lett., 62, 539-549.

V. Andrieu, L. Praly and A. Astolfi. (2009). High gain observers with updated high-gain and homogeneous correction terms, Automatica, 45(2), 422-428.

M. Arcak and D. Nešić. (2004). A framework for nonlinear sampled-data observer design via approximate discrete-time models and emulation, Automatica, 40, 1931-1938.

J. P. Barbot, S. Monaco, and D. Normand-Cyrot. (1999). Discrete-time approximated linearization of SISO systems under output feedback, IEEE Trans. Automat. Contr., 44(9), 1729-1733.

D. Bestle and M. Zeitz. (1983). Canonical form observer design for nonlinear time variable systems, Int. J. Contr., 38(2), 419-431.

F. Deza. (1991). Contribution to the synthesis of exponential observers, PH. D. Dissertation, INSA Rouen, France.

F. Deza, E. Busvelle, J. P. Gauthier and D. Rakotopora. (1992). High gain estimation for nonlinear systems, Syst. Control Lett., 18, 295-299.

F. Deza, D. Bossanne, E. Busvelle, J. P. Gauthier and D. Rakotopara. (1993). Exponential observers for nonlinear systems, IEEE Trans. Automat. Contr., 38(3), 482-484.

J. P. Gauthier, H. Hammouri and S. Othman. (1992). A simple observer for nonlinear systems applications to bioreactors, IEEE Trans. Automat. Contr., 37(6), 875-880.

J. P. Gauthier and I. A. Kupka. (1994). Observability and observers for nonlinear systems, SIAM Journal Contr. and Optimiz., 32(4), 975-994.

K. Gu. (2000). An integral inequality in the stability problem of time-delay systems. In Proceeding of 39th IEEE Conference on Decision and Control, Sydney, Australia, 2805-2810.

I. Haskara, Ü. Özgüner and V. Utkin. (1998). On sliding mode observers via equivalent control approach, Int. J. Contr., 71(6), 1051-1067.

I. Karafyllis and C. Kravaris. (2009). From continuous-time design to sampled-data design of observers, IEEE Trans. Automat. Contr., 54(9), 2169-2174.

I. Karafyllis. (2007). A system-theoretic framework for a wide class of systems I: Applications to numerical analysis, $J$. Math. Anal. 328, 876-899.

I. Karafyllis. (2007). A system-theoretic framework for a wide class of systems II: Input-to-output stability, J. Math. Anal., 328, 466-486.

I. Karafyllis and Z.-.P Jiang. (2007). A small-gain theorem for a wide class of feedback systems with control applications, SIAM Journal Contr. and Optimiz., 46(4), 1483-1517.

H. K. Khalil. (2004). Performance recovery under output feedback sampled-data stabilization of a class of nonlinear systems, IEEE Trans. Automat. Contr., 49(12), 2173-2184.
A. J. Krener and A. Isidori. (1983). Linearization by output injection and nonlinear observers, Syst. Control Lett., 3(1), $47-52$

Y. Li, X. Xia and Y. Shen. (2013). A high-gain-based global finite-time nonlinear observer, Int. J. Contr., 86(5), 759-767.

M. Mahmoud and H. Khalil. (2002). Robustness of high-gain observer-based nonlinear controllers to unmodeled actuators and sensors. Automatica, 38(2), 361-369.

H. Michalska and D. Mayne. (1995). Moving horizon observers and observer-based control, IEEE Trans. Automat. Contr., 40(6), 995-1006.

M. Nadri, H. Hammouri and R. M. Grajales. (2013). Observer design for uniformly observable systems with sampled measurements, IEEE Trans. Automat. Contr., 58(3), 757762 .

D. Nešić, and A. R. Teel. (2004). A framework for stabilization of nonlinear sampled-data systems based on their approximate discrete-time model, IEEE Trans. Automat. Contr., 49(7), 1103-1122.

D. Nešić, A. R. Teel and P. V. Kokotović. (1999). Sufficient conditions for stabilization of sampled-data nonlinear systems via discrete-time approximations, Syst. Control Lett., 38, 259-270.

R. Postoyan and D. Nešić. (2012). On emulated nonlinear reduced-order observers for networked control systems, Automatica, 48, 645-652.

L. Praly. (2003). Asymptotic stabilization via output feedback for lower triangular systems with output dependent incremental rate, IEEE Trans. Automat. Contr., 48(6), 1103-1108.

T. Raff, M. Kögel and F. Allgöwer. (2008). Observer with sampleand-hold updating for lipschitz nonlinear systems with nonuniformly sampled measurements, in ACC'08 (American Control Conference), Seattle, Washington, USA.

S. Raghavan and J. K. Hedrick. (1994). Observer design for a class of nonlinear systtems, Int. J. Contr., 59(2), 515-528.

J. Rudolph and M. Zeitz. (1994). A block triangular nonlinear observer normal form, Syst. Control Lett., 23(1), 1-8.

Y. Shen and X. Xia. (2008). Semi-global finite-time observers for nonlinear systems, Automatica, 44(12), 3152-3156.

Y. Shen and Y. Huang. (2009). Uniformly observable and globally lipschitzian nonlinear systems admit global finitetime observers, IEEE Trans. Automat. Contr., 55(11), 9951006.

H. Shim, Y. I. Son and J. H. Seo. (2001). Semi-global observer for multi-output nonlinear systems, Syst. Control Lett., 42, 233-244.

F. E. Thau. (1973). Observing the state of nonlinear dynamic systems, Int. J. Contr., 17(3), 471-479.

V. Van Assche, T. Ahmed-Ali, C. Ham and F. LamnabhiLagarrigue. (2011). High gain observer design for nonlinear systems with time varying delayed measurements, in 18th IFAC World Congress, Milan, Italy.

W. Wang, D. Nešić and R. Postoyan. (2015). Emulation-based stabilization of networked control systems implemented on FlexRay, Automatica, 59, 73-83.

X. Xia and W. Gao. (1988). On exponential observers for nonlinear systems, Syst. Control Lett., 17(4), 319-325.

X. Xia and W. Gao. (1989). Nonlinear observer design by observer error linearization, SIAM Journal Contr. and Optimiz., $27(1), 199-216$.

M. Zeitz. (1987). The extended Luenberger observer for nonlinear systems, Syst. Control Lett., 9(2), 149-156.

D. Zhang, Y. Shen and X. Xia. (2014). Continuous observer design for nonlinear systems with sampled and delayed output measurements, in 19th IFAC World Congress, Cape Town, South Africa, 269-274. 